\title{
Kynurensäure und Xanthurensäure bei Habrobracon juglandis Ashmead
}

\author{
Friedrich Leibenguth
}

\author{
Entwicklungsphysiologische Abteilung (Leiter: Prof. Dr. V. Schwartz) \\ des Zoologischen Instituts der Universität Tübingen \\ (Z. Naturforschg. 20 b, 315-317 [1965] ; eingegangen am 6. Oktober 1964)
}

Herrn Prof. Dr. Alfred Kühn in Verehrung zum 80. Geburtstag gewidmet

\begin{abstract}
Bis zum Einsetzen der Ommochromsynthese bei Habrobracon werden die Vorstufen Kynurenin und 3-Hydroxykynurenin zu Kynurensäure und Xanthurensäure umaminiert. Vom Vorpuppenstadium ab ist dieser seitliche Abbauweg stillgelegt. Ursache kann nicht Substratmangel sein; es wird vielmehr Verlust der Transaminase-Aktivitäten angenommen.
\end{abstract}

Durch die biochemische Analyse der Augenfarbmutanten von Drosophila und Ephestia ist der Mechanismus der Ommochromsynthese, der von der Aminosäure Tryptophan seinen Ausgang nimmt und über Kynurenin und 3-Hydroxykynurenin verläuft, im wesentlichen geklärt worden ${ }^{1}$.

(im Säuger: Anthranilsäure $\underset{\uparrow}{3 \text {-Hydroxyanthranilsäure) }}$

Tryptophan $\rightarrow$ Kynurenin $\rightarrow 3$-Hydroxykynurenin $\rightarrow$ Ommochrome Kynurensäure Xanthurensäure

Der neue Schwerpunkt genphysiologischer Forschung, die Frage nach differentieller Aktivierung der für die Enzyme dieser Wirkkette verantwortlichen Gene in der postembryonalen Entwicklung kann nur angegangen werden, wenn alle SyntheseZwischenstufen und -Endprodukte erfaßt werden. In vielen Fällen werden quantitativen stadienspezifischen Unterschieden bestimmter Produkte gleiche Fermentaktivitäten zugrunde liegen. Die Menge eines Zwischenproduktes wird dann geregelt durch das Substratangebot und die Nachfrage durch eine weiterführende Reaktion. Es lassen sich aber auch in besonderen Fällen, wenn ein Endprodukt vorliegt, dessen Vorläufer quantitativ bestimmbar ist, Rückschlüsse auf unterschiedliche Fermentaktivität ziehen.

Ein solcher Fall liegt vor bei den Umsetzungen Kynurenin $\rightarrow$ Kynurensäure (Ksr) und 3-Hydroxykynurenin $\rightarrow$ Xanthurensäure (Xsr) bei Habrobracon $^{*}$, die damit erstmals für ein Hymenopter nachgewiesen werden, nachdem Kynurensäure ${ }^{2}$ und Xanthurensäure ${ }^{3}$ schon bei Drosophila, letztere auch bei Plodia ${ }^{4}$ und Ephestia ${ }^{5}$ gefunden worden waren.

1 Zusammenfassung: I. Ziegler, Adv. Genet. 10, 349 [1961].

* Frau Dr. Anna R. Whiting und Herrn Dr. R. C. von Borstel danke ich für die Zusendung der Tiere.

2 R. Danneel u. B. Zimmermann, Z. Naturforschg. 9 b, 788 [1954].

\section{Material und Methodik}

Die Zucht von Habrobracon erfolgte an ausgewachsenen Raupen der a-Mutante von Ephestia kühniella bei $30{ }^{\circ} \mathrm{C}$ in Glasschalen mit flachem Deckel. Die Entwicklung dauert 10 Tage. Die aus dem Ei schlüpfende Larve saugt an der Wirtsraupe und wird nach 3 Tagen als alte Freßlarve bezeichnet $\left(\mathrm{Fl}_{3}\right)$. Sie wandert vom Wirt weg und spinnt einen Kokon (Spinnlarve, Sl). Am 5. Tag häutet sie sich zur Vorpuppe (Vp). Am 6. Tag liegt die junge weiße Puppe mit braunrötlichen Augen vor $\left(\mathrm{P}_{1}\right)$. Die Augen sind am 7. Tag tiefbraun $\left(\mathrm{P}_{2}\right)$ und werden gleichzeitig mit der Körperausfärbung am Tag darauf braunschwarz $\left(\mathrm{P}_{3}\right)$. Zwischen dem 9 . und 10. Tag schlüpft die Imago (I). Bei der Vorpuppenhäutung wird ein erstes Exkret abgegeben, bei der Imaginalhäutung ein zweites.

Je 10 Tiere wurden zur Extraktion in Glasröhrchen mit $0,2 \mathrm{ml}$ eines Extraktionsgemisches (Methanol : $1 \%$ $\mathrm{NH}_{3}=3: 1$ ) übergossen und mit einem Glasstab fein zerrieben. Nach Zentrifugierung $(3600 \mathrm{U} / \mathrm{min})$ wurde die überstehende Flüssigkeit auf Chromatographierpapier (Schleicher \& Schüll $2043 \mathrm{~b}$ ) aufgetragen. Die Trennung erfolgte zweidimensional in 70-proz. Propanol und 4-proz. Na-Citrat. Die unter der UV-Lampe $(365 \mathrm{~m} \mu)$ an ihrer Fluoreszenz kenntlichen Kynurensäure- und Xanthurensäureflecke wurden mit Bleistift umrandet, ausgeschnitten und fluorometriert ${ }^{5}$. Aus den Fluoreszenzwerten von in 4-proz. Na-Citrat gelaufenen Eichchromatogrammen mit den synthetischen Substanzen (Schuchardt) wurden Eichkurven zusammengestellt, die bis zu Konzentrationen von $1 \gamma$ (Ksr) bzw. $2 \gamma$ (Xsr) linear verlaufen.

Die Identifizierung von Kynurensäure und Xanthurensäure erfolgte durch Vergleich ihrer $R_{f}$-Werte mit denen der synthetischen Substanzen in verschiedenen Laufmitteln sowie durch Farbreaktionen (Xsr) ${ }^{4}$ und photochemisches Verhalten $(\mathrm{Ksr})^{6}$. Außerdem wurden die UV-Spektren (Zeiss-Spektralphotometer)

\footnotetext{
3 Y. Umebachi u. K. Tsuchitani, J. Biochemistry [Tokyo] 42, 817 [1955].

4 E. Möhlmann, Z. Vererbl. 89, 651 [1958].

5 A. Egelhata, Z. Vererbl. 94, 349 [1963].

6 O. Wiss u. H. Hellmann, Z. Naturforschg. 8 b, 70 [1953].
} 
aufgenommen, die in der Lage der Maxima mit den Spektren der synthetischen Substanzen übereinstimmen.

$\lambda_{\max }\left(\mathrm{H}_{2} \mathrm{O}\right)$

$\begin{array}{ll}\text { Kynurensäure } & 245,330 \mathrm{~m} \mu, \\ \text { Xanthurensäure } & 240,340 \mathrm{~m} \mu .\end{array}$

\section{Ergebnisse und ihre Diskussion}

Die Bildung von Anthranilsäure und 3-Hydroxyanthranilsäure durch Eliminierung der Seitenkette bei Kynurenin und 3-Hydroxykynurenin - für den Säugerorganismus beschrieben - wurde bei Habrobracon nicht festgestellt. Hingegen dürfte der Mechanismus der Kynurensäure- und Xanthurensäurebildung hier wie dort der gleiche sein (Umaminierung) ${ }^{7}$.

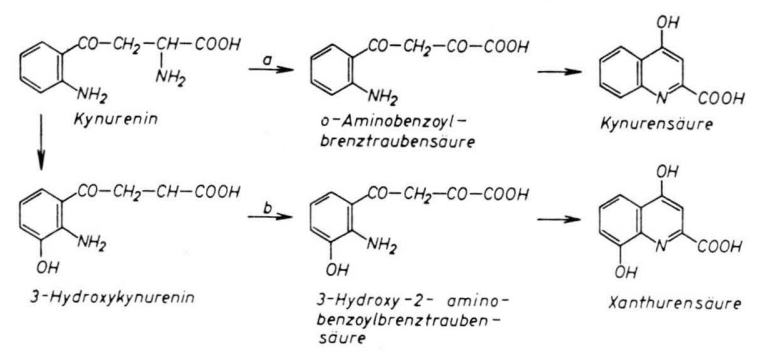

Es steht nicht fest, ob an Reaktion a und b zwei verschiedene Transaminasen beteiligt sind. Jedenfalls werden in beiden Fällen Pyridoxal-5-phosphat als Coenzym und eine $\alpha$-Ketosäure benötigt. Offenbar erfolgt der spontane Ringschluß sehr rasch, denn sowohl $o$-Aminobenzoylbrenztraubensäure als auch 3-Hydroxy2-amino-benzoylbrenztraubensäure konnten bei Habrobracon nicht isoliert werden.

Xanthurensäure wird bei Habrobracon erstmals in zwei Tage alten Freßlarven gefunden (10 Tiere/Bestimmung), während Kynurensäure unmittelbar vor dem Spinnlarvenstadium auftritt. Beide Substanzen bleiben in der weiteren Entwicklung stets nachweisbar (Abb. 1). Der signifikante Gipfel der Xanthurensäure-Konzentration während des Spinnlarvenstadiums $\left(\bar{x}_{\mathrm{SI}}-\bar{x}_{\mathrm{Fl}_{3}}>\right.$ $3,27 \cdot s_{\text {Diff }}$ für $0,1 \%$ Überschreitungs-Wahrscheinlichkeit) kommt deshalb so deutlich zum Ausdruck, weil 85\% dieses Endproduktes mit dem Erstexkret ausgeschieden werden. Das trifft auch für die Kynurensäure zu, deren höhere Konzentration auf dem Spinnlarvenstadium sich jedoch nicht gegenüber der der Vorpuppen absichern läßt (Warngrenze $=5 \%$ ). Die Konzentration beider Substanzen bleibt während des Puppenstadiums konstant $(0,1 \gamma / 10$ Tiere $)$. Bei der Imaginalhäutung erfolgt die Ausscheidung.

Die Bildung der Endprodukte Kynurensäure und Xanthurensäure ist abhängig von der Transaminase-

\footnotetext{
7 O. WIss, Hoppe-Seyler's Z. physiol. Chem. 293 [1953].
}

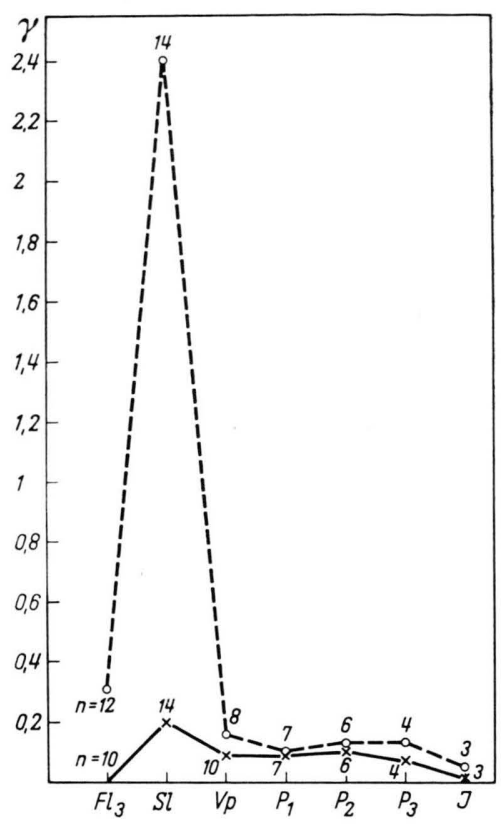

Abb. 1. Kynurensäure- und Xanthurensäure-Konzentration/ 10 Tiere während der Entwicklung. Mittelwerte aus der jeweils angegebenen Anzahl Messungen. —_: Kynurensäure, - - : Xanthurensäure.

Aktivität und dem Angebot an Vorstufen. 3-Hydroxykynurenin als Vorstufe von Xanthurensäure wird erst vom Vorpuppenstadium ab zur Ommochromsynthese verwendet und kann bis zu diesem Zeitpunkt zu Xanthurensäure umaminiert werden. Die Schlußfolgerung, daß auf dem Puppenstadium deshalb keine Xanthurensäure gebildet wird, weil die Ommochromsynthese der Transaminase das Substrat entzieht, trifft jedoch nicht zu. Denn auf Stadium $\mathrm{P}_{2}$ ist die 3-Hydroxykynurenin-Konzentration mit $8-10 \gamma / 10$ Tiere mindestens doppelt so hoch wie auf dem Spinnlarvenstadium ${ }^{8}$. Die Tatsache, daß Xanthurensäure und 3-Hydroxykynurenin bis zum Spinnlarvenstadium im Fettkörper und Hämolymphe gefunden werden, wo letztere Substanz auch während des Puppenstadiums vorkommt, schließt eine Änderung der lokalen Beziehung zwischen Enzym und Substrat aus.

Dies trifft auch für die Kynurensäurebildung zu. Kynurenin dürfte jedoch bis zum Spinnlarvenstadium der Transaminierung weitgehend dadurch entzogen sein, daß es in vivo rasch zu 3-Hydroxykynurenin umgesetzt wird. Wenn diese Annahme stimmt, müßte durch Erhöhung des Kynureninspiegels bei den Freßund Spinnlarven auf dem Spinnlarvenstadium eine ähnlich erhöhte Kynurensäure-Ausbeute zu erzielen sein, wie sie bei der Xanthurensäure vorliegt. Um der Kynurenin-Transaminase mehr Substrat zu bieten, stehen zwei Möglichkeiten zur Verfügung. Der genetische Block zwischen Kynurenin und 3-Hydroxykynure-

\footnotetext{
8 In Vorbereitung.
} 
nin bei der orangeäugigen Mutante (o) führt zu einer Anreicherung von Kynurenin (5 $\gamma / 10$ Spinnlarven gegegenüber $1 \gamma$ bei $o^{+}$). Die zweite Möglichkeit besteht darin, Tryptophan an die Freßlarven der Wildform zu verfüttern (Injektion von je $10 \mu \mathrm{l}$ einer L-Tryptophansuspension in die a-Ephestia-Wirtsraupen). In beiden Fällen hat der erhöhte Kynureninspiegel eine signifikant gesteigerte Kynurensäure-Produktion zur Folge $(>1,4 \gamma / 10$ Spinnlarven der Mutante, $2 \gamma / 10$ Spinnlarven der Wildform nach Tryptophan-Verfütterung). Wie bei der Xanthurensäure bleibt auch der Gipfel der Kynurensäure-Konzentration auf das Spinnlarvenstadium beschränkt. Während des Puppenstadiums haben Mutante, Tryptophan-gefütterte und intakte Wildform einen gleichniedrigen Kynurensäurespiegel von $0,1 \gamma /$ 10 Tiere. Das ist um so bemerkenswerter, als die Mutante während dieser Phase der Entwicklung eine Kynurenin-Konzentration von $10 \gamma / 10$ Tiere aufweist. Substratmangel kann also für die fehlende Kynurensäure-Synthese nicht verantwortlich gemacht werden. Schon Spuren von Transaminase-Aktivität müßten zu einer meßbaren Verschiebung der Konzentrations-Verhältnisse führen.

Für Mormoniella werden zwei Substanzen beschrieben, die das photochemische Verhalten von Kynurensäure zeigen ${ }^{9}$. Sie sind im Larvendarm vorhanden, verschwinden infolge Ausscheidung und treten während des Puppenstadiums wieder auf. Das vollständige Ver-

${ }^{9}$ G. B. S $\mathrm{AUL}, 2$ nd, Rev. suisse Zool. 67, 270 [1960]. schwinden deckt sich nicht mit den vorliegenden an Habrobracon gewonnenen Ergebnissen.

Um zu dem eingangs zusammengefaßten Bild von den Umsetzungen Kynurenin $\rightarrow$ Kynurensäure und 3-Hydroxykynurenin $\rightarrow$ Xanthurensäure zu kommen, muß folgendes vorausgesetzt werden:

1. Es handelt sich um zwei verschiedene Transaminasen. Würden beide Schritte von einem Enzym katalysiert, so könnte sich die weitere Untersuchung auf eine Umsetzung beschränken.

2. Für beide Transaminasen sind Strukturgene vorhanden ${ }^{10}$.

3. Coenzym und $\alpha$-Ketosäure sind während der postembryonalen Entwicklung in konstanter Konzentration vorhanden.

Treffen diese Voraussetzungen zu, dann rechtfertigen die aufgezeigten Befunde die Annahme, daß bei Habrobracon mit Beginn der Metamorphose eine Umsteuerung im Tryptophanstoffwechsel erfolgt. Die endgültige Klärung kann nur durch den enzymatischen Test in vitro erbracht werden. Darüber hinaus bleiben noch viele Probleme (Umsteuerungsmechanismus; Zugehörigkeit der beiden Strukturgene zum gleichen Operon?), an die heranzuführen, Zweck dieser Mitteilung ist. Die Arbeit wird fortgesetzt und in erweitertem Zusammenhang an anderer Stelle publiziert.

10 H. S. Forrest, Proc. XI Int. Congr. Genetics Vol. I, S. 4, 1963. 